\title{
Radiation oncology health technology assessment - the best is the enemy of the good
}

\author{
Søren M Bentzen
}

Several radiation technologies are becoming available, such as image-guided radiation therapy and proton and carbon ion therapy, but most come with a heavy price tag. A new proton therapy center, for example, can cost $\$ 100$ 140 million (New York Times, December 26, 2007). With general health-care costs spiraling out of control, the issues of health technology assessment and cost-benefit can no longer be ignored.

An interesting asymmetry exists between getting approval for a drug compared with a medical device. The FDA approves a new drug for a given medical indication based on evidence from randomized controlled trials that the drug provides a net benefit over standard therapies. By contrast, medical devices can be marketed with a so-called 510(k) FDA approval; in essence a certification that states that the device does what it is meant to do and that using it does not compromise patient safety. Critics argue that a new technology is 'just another drug' and, therefore, the benefit of any new device should be demonstrated in randomized controlled trials before FDA approval.

There is merit to insisting that if a new technology is claimed to improve treatment outcome, this should be supported by evidence from a randomized trial. However, a new technology might improve treatment quality without a measurable impact on clinical outcome because of the low sensitivity and specificity of clinical endpoints (Bentzen S [2008] Radiother Oncol 86: 142-147).

Purists maintain that new technologies should be introduced into clinical practice only if a survival advantage has been proven. Yet, most people would probably hesitate to use a new technology that yielded poorer treatment quality. Remember how difficult it was to arrive at a consensus that postoperative radiation therapy improves survival of patients with breast cancer? Imagine a new, cheaper device for breast radiotherapy that causes, for example, $10 \%$ underdosage of part of the breast tissue. A rough ....if we insist

on the 'best', namely

randomized

comparisons

of treatment

outcome

from new

technologies... we will

continue to

miss out on the

'good'...

SM Bentzen is

Professor of Human

Oncology at the

University of

Wisconsin School

of Medicine and

Public Health,

Madison, WI, USA.

Competing interests

The author declared no

competing interests.

www.nature.com/clinicalpractice doi:10.1038/ncponc1203 estimate, based on the results of randomized trials of surgery with or without adjuvant radiotherapy, is that this device would result in a loss of $<1 \%$ in 10 -year survival. By use of standard design parameters, a randomized trial intended to detect this survival detriment would need to accrue more than 90,000 patients. Would the fact that this trial would almost certainly never be conducted make this new device acceptable to physicians and patients?

Much of the technology discussion relates to health economics. While a cost-benefit comparison requires a proven differential outcome, a cost-utility analysis compares alternative technologies. A naïve example of a cost-utility analysis would involve the comparison of two competing technologies that improve the geometrical precision of radiation therapy, thereby reducing the margin of normal tissue that needs to be irradiated. In this case, we would compare the cost in monetary units per millimeter reduction of the required dosimetric margin.

Voltaire, in his Dictionnaire Philosophique (1764), wrote that "The best is the enemy of the good" - an apparent paradox and often misquoted. Maybe in the case of health technology assessment, Voltaire was right; if we insist on the 'best', namely randomized comparisons of treatment outcome from new technologies - a bar raised so high that in practice we rarely reach it - we will continue to miss out on the 'good', namely critical, systematic comparisons of technologies and devises in terms of operational or quality criteria.

Introduction of a new technology into the setting of a prospective clinical trial with rigorous quality control, patient follow-up and analysis of outcome would undoubtedly produce useful data regarding the clinical value of this technology. Radiation oncologists must engage in the development of novel paradigms for critical health technology assessments without the ideal requirement of randomization. Let us start doing what we can, rather than continuing to wait for Godot! 